\title{
Content-Based Image Retrieval Using Customized Convolutional Neural Network
}

\author{
A.P. Nilawar ${ }^{1}$, C. G. Dethe ${ }^{2}$, A. Jaiswal ${ }^{3}$ and J. D. Kene ${ }^{4}$ \\ ${ }^{1,3,4}$ Assistant Professor, Department of Electronics and Communication Engineering, Shri \\ Ramdeobaba College of Engineering and Management, Nagpur, Maharashtra, India \\ ${ }^{2}$ Director, UGC HRDC, R.T.M.N.U., Nagpur, Maharashtra, India
}

\section{ABSTRACT}

In today's world due to multimedia development, there is a huge image database. Content-Based Image retrieval (CBIR) is a widely used method for image retrieval from a large image database. Existing retrieval methods are based on the basic content of an image like color, Shape, and Texture. The system based on basic features requires more time for processing and provides less accuracy. To reduce time and improve accuracy we are proposing CBIR Using CNN in this paper. CNN is used for feature extraction and similarity measurement Hamming distance is used. In this technique, the user has to provide an image as an input query image. The similar images related to the query image are displayed as a result. The performances of a system are evaluated by precision and mean average precision (MAP). After comparing with existing methods, we found encouraging results that lead to improving accuracy.

KEY WORDS: CONTENT-BASED IMAGE RETRIEVAL, CNN, DNN, LSH.

\section{INTRODUCTION}

In this day and age, there is an expansion in the component of interactive media databases. It comprises information like image, sound, video, and so forth. The utilization of images in the area of medicinal, individual, news coverage makes a lot of image databases. In this manner, the Content-Based Image Retrieval system was created to manage huge image databases. Content-Based Image retrieval system restores a lot of images from a progression of images in the database to fulfill the user's need with closeness assessment. The CBIR framework has two fundamental stages in the primary stage: the pre-processing with feature extraction dependent on

\section{ARTICLE INFORMATION}

*Corresponding Author: aa.nilawar@gmail.com

Received 14th Oct 2020 Accepted after revision 29th Dec 2020

Print ISSN: 0974-6455 Online ISSN: 2321-4007 CODEN: BBRCBA

Thomson Reuters ISI Web of Science Clarivate Analytics USA and Crossref Indexed Journal

\section{Clarivate crossef}

NAAS Journal Score 2020 (4.31)

A Society of Science and Nature Publication,

Bhopal India 2020. All rights reserved.

Online Contents Available at: http//www.bbrc.in/

Doi: $h t t p: / / d x$.doi.org/10.21786/bbrc/13.14/105 attributes such as color, shape, and texture. In the second stage assessment of similarity between a query image and indexed images from the database is performed.

Figure 1: Basic CBIR System

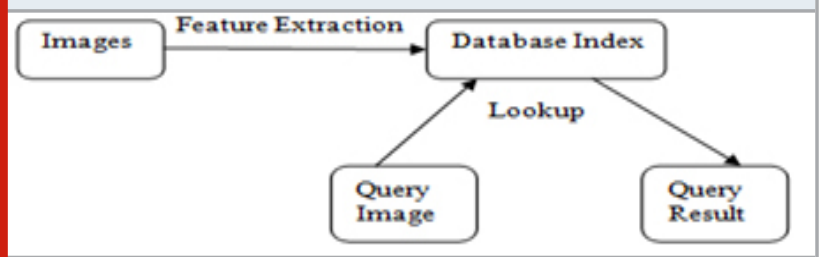

The above figure explains the basic operation of the CBIR System. In the retrieval system, there are essentially three most important fields: text-based image retrieval, retrieval based on visual features, and semantic features. In a textbased image retrieval system images are provided through labels and for retrieval cause similarity of these labels are used. A predominant disadvantage of these types of structures is that a lot of human work evolved for image 
labeling and if the database of images in a massive quantity then it is pretty difficult. And labeling of images is no longer user friendly; it relies upon database creators labeling strategies. Then the visual-based image retrieval systems used for image retrieval purposes and lowlevel features are considered for feature extraction. The systems are based on visual features along with semantic features in use. The three most important features that exist in an image specifically are color, texture, and shape (Latif, A et. al. and Ganar, A.N et. al.). All the methods utilizing color, text, and shape features are time-consuming because for each feature we require to train the image database then compute the results. Hence CNN is used for feature extraction. Once the pre-training is done it will provide faster results.

\section{Literature Survey}

2.1 Neural Network: Neural Network is an interconnected network of neurons in which inputs are independent variables and output is dependent on the weighted inputs connected to neurons. The neural network consists of input values, weights, activation function, and output values (LeCun, Y). Generally, four activation functions are commonly used: Threshold function, Sigmoid function, Rectifier function, and Hyperbolic Tangent function. Depending on the application appropriate activation functions can be selected. The weights are estimated by using Gradient Descent and Back Propagation algorithm. The weighted inputs are applied to a neuron to get the output. And inside the neuron activation function is applied to the weighted sum of the inputs present. The output is determined by using initial weights that can be random or fixed. Finally, an error is calculated and a Backpropagation algorithm is applied to fine-tune the weights and reduce the error.

2.2 Convolutional Neural Network: The Convolutional Neural Network is a neural network having four steps. The first step is applying 2D convolution to the input image. 2D Convolution is a mathematical operation in which two matrices are used. For the application of CNN one matrix is an image and the other matrix is a feature detector. Generally, the Feature detector is a matrix of size $3 \times 3$ but size may change. In a convolution operation, when a feature detector is applied to an image, a matrix with different values from the original is obtained $(\mathrm{Wu}$, $\mathrm{J})$. In this step, the feature detector is applied to the input image which results in the generation of the feature map. For removing unwanted information in the feature map, the ReLU (Rectified Linear Unit) activation function is used (Kuo, C.C.J). In the second stage, the pooling operation is performed. Three types of pooling are used: max pooling, mean pooling, and sum pooling. Stage three consists of flattening, which generates a feature map. And in the last stage full connection is done. The softmax and cross-entropy are the two functions used for improving accuracy in CNN.
Figure 2: Basic Convolutional Neural Network

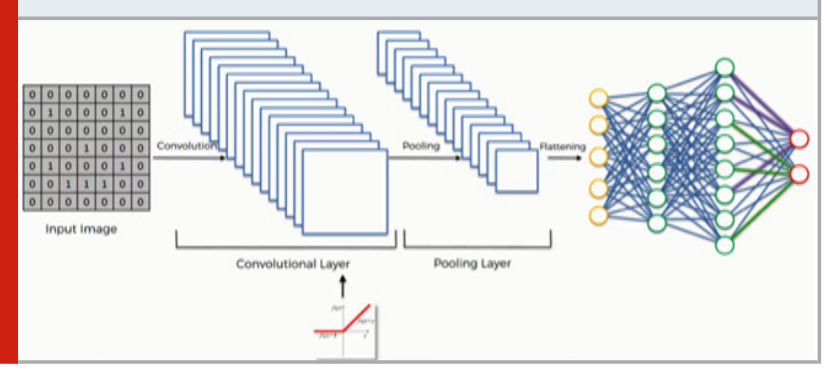

The above figure 2 shows Basic Convolutional Neural Network which is used for feature extraction. In this CNN, max-pooling, and ReLU are used. The input for this network is an image. The output is provided by a fully connected CNN network (Simonyan, K. and Zisserman, A).

\section{Proposed Method}

Figure 3: Proposed Method

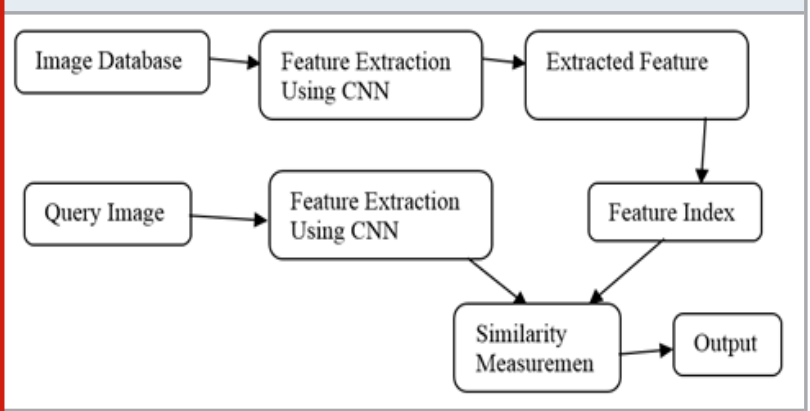

Figure 3 provides the proposed method of data flow. The algorithm for the proposed method is explained below. Step 1: In an image database several images with different categories are present.

Step 2: These images are fed to CNN for feature extraction.

Step 3: For each image feature vector generated. Each vector consists of 1280 elements.

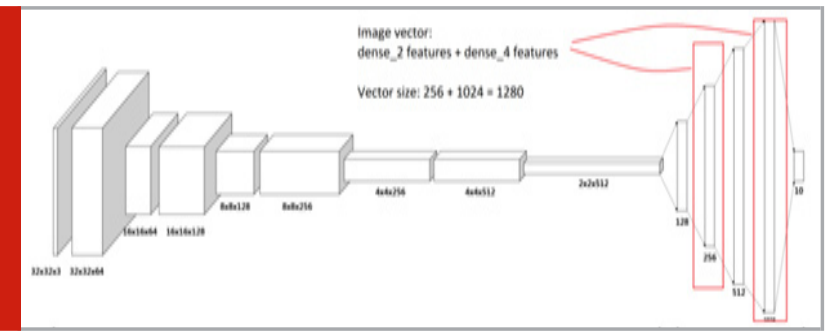

Step 4: Features are indexed in the database.

Step 6: The user has to provide an input image considered as a query image.

Step 7: Features of a query image extracted using CNN.

Step 8: Using Hamming distance similarity between features of the query image matched with the index. 


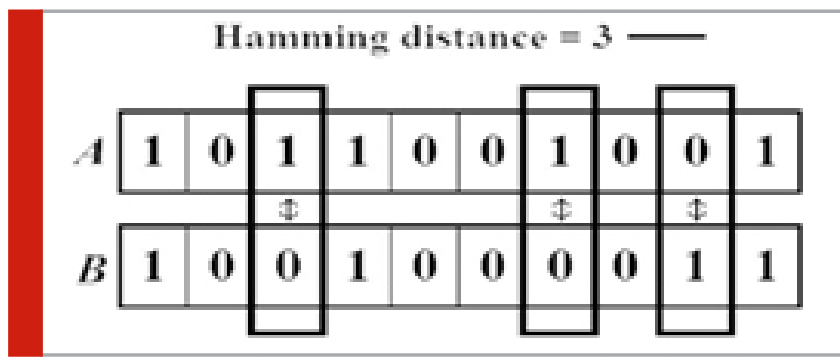

Step 9: Finally similar images are displayed.

Step 10: Repeat from step 6 for another input.

Performance Evaluation: The performance of the system was evaluated using two parameters Precision and Mean Average Precision (MAP). Precision is the ratio of the number of relevant images retrieved to the total number of images retrieved.

$$
\mathrm{MAP}=\sum_{k}^{K} \frac{\mathrm{Avg} \mathrm{P}(\mathrm{k})}{\mathrm{K}}
$$

Where $\mathrm{k}=$ value of precision for query and $\mathrm{K}$ is the number of query images.

\section{EXPERIMENTAL RESULTS AND DISCUSSION}

The image database consists of 60000 images with 10 categories (CIFAR Image Database). The performance of $\mathrm{CNN}$ is given in figure 4.

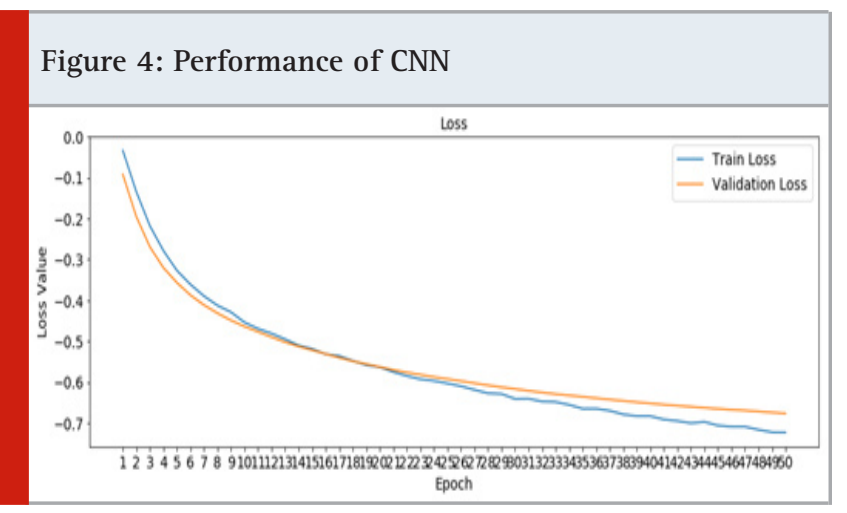

After training the model we evaluate the performance of the system by providing a query image. The results show the matched images from the image database. Figure 5 shows sample results that are tested on a few query images.

The database consists of 10 categories and in each category 6000 images. Figure 5 shows sample results of some categories like airplanes, cats, and birds. The top 10 matching images are considered for performance evaluation.

Table 1 represents the performance of the proposed system with precision and MAP. We can conclude that
Figure 5: Sample Results of the proposed system

Query image

es

Result images

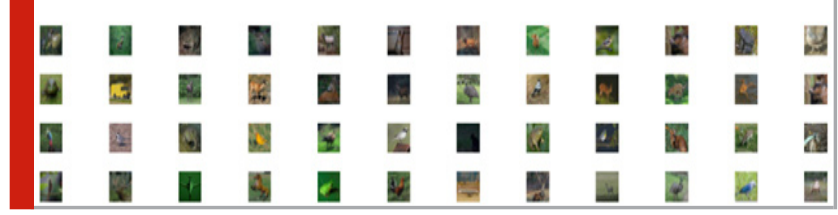

Table 1. Precision and MAP

\begin{tabular}{|l|c|}
\hline Category & Precision \\
\hline Airplane & 90.18 \\
\hline Automobile & 90.28 \\
\hline Bird & 90.28 \\
\hline Cat & 90.70 \\
\hline Dear & 90.70 \\
\hline Dog & 90.24 \\
\hline Frog & 90.20 \\
\hline Horse & 90.25 \\
\hline Ship & 90.90 \\
\hline Truck & 90.85 \\
\hline MAP & 90.45 \\
\hline
\end{tabular}

Table 2. Comparisons with existing methods

\begin{tabular}{|l|c|c|}
\hline Methods & $\begin{array}{c}\text { Images in } \\
\text { Database }\end{array}$ & $\begin{array}{c}\text { Precision } \\
\%\end{array}$ \\
\hline $\begin{array}{l}\text { Color in YCBCR, Color } \\
\text { Moment (Dandotiya, } \\
\text { Y. and Atre, A) }\end{array}$ & 1000 Images & 82.70 \\
\hline $\begin{array}{l}\text { HSV Color Histogram and Hu } \\
\text { moment invariant (Rajkumar, } \\
\text { R. and Sudhamani, D.M) }\end{array}$ & 1000 Images & 84.20 \\
\hline $\begin{array}{l}\text { CBIR Using Customised } \\
\text { CNN (Proposed) }\end{array}$ & 60000 Images & 90.45 \\
\hline
\end{tabular}

in all categories results provides promising results. Table 2 provides a compression analysis with existing methods.

\section{CONCLUSION AND FUTURE WORK}

We can conclude that the performance of CBIR improves significantly using CNN. Since the existing methods based on image content like color, shape, and texture provides less accuracy as compared to the proposed system. We can use CNN for improving accuracy and retrieval time. In all categories presented in the database, the proposed 
system provides significant results. In the future, we can evaluate CNN using different image databases.

\section{REFERENCES}

Dandotiya, Y. and Atre, A., 2017, April. Image retrieval using edge detection, RLBP, color moment method for YCbCr and HSV color space. In 2017 International conference of Electronics, Communication and Aerospace Technology (ICECA) (Vol. 2, pp. 662-668). IEEE.

Ganar, A.N., Gode, C.S. and Jambhulkar, S.M., 2014, January. Enhancement of image retrieval by using colour, texture and shape features. In 2014 International Conference on Electronic Systems, Signal Processing and Computing Technologies (pp. 251-255). IEEE.

Image Database https://www.cs.toronto.edu/ kriz/cifar. html

Kuo, C.C.J., 2016. Understanding convolutional neural networks with a mathematical model. Journal of Visual
Communication and Image Representation, 41, pp.406413.

Latif, A., Rasheed, A., Sajid, U., Ahmed, J., Ali, N., Ratyal, N.I., Zafar, B., Dar, S.H., Sajid, M. and Khalil, T., 2019. Content-based image retrieval and feature extraction: a comprehensive review. Mathematical Problems in Engineering, 2019.

LeCun, Y., 1998. The MNIST database of handwritten digits. http://yann. lecun. com/exdb/mnist/.

Rajkumar, R. and Sudhamani, D.M., 2019. Development of retrieval system using color from multi-segment and shape features from an image as a combination. International Journal of Engineering and Technology, 9(1).

Simonyan, K. and Zisserman, A., 2014. Very deep convolutional networks for large-scale image recognition. arXiv preprint arXiv:1409.1556.

$\mathrm{Wu}$, J., 2017. Introduction to convolutional neural networks. National Key Lab for Novel Software Technology. Nanjing University. China, 5, p.23. 